\title{
Expression of Gli1 in the hedgehog signaling pathway and breast cancer recurrence
}

\author{
Naoko Takebe ${ }^{1}$, Sally Hunsberger ${ }^{2}$, Sherry X. Yang ${ }^{3}$ \\ ${ }^{1}$ Cancer Therapy Evaluation Program, ${ }^{2}$ Bioinformatics Research Branch, and ${ }^{3}$ National Clinical Target Validation Laboratory, Division of Cancer \\ Treatment and Diagnosis, National Cancer Institute, National Institutes of Health, USA \\ Corresponding to: Naoko Takebe, MD, PhD. Cancer Therapy Evaluation Program, 6130 Executive Blvd, EPN7124, Rockville MD 20852, USA. \\ Email: takeben@mail.nih.gov; Sherry X. Yang, MD, PhD. National Clinical Target Validation Laboratory, 37 Convent Drive, Bldg.37/Rm. 1048, \\ Bethesda, MD 20892, USA. Email: Sherry.Yang@nih.gov.
}

Submitted Aug 27, 2012. Accepted for publication Sep 28, 2012.

DOI: $10.3978 /$ j.issn.1000-9604.2012.09.07

Scan to your mobile device or view this article at: http://www.thecjcr.org/article/view/1107/1506

The hedgehog $(\mathrm{Hh})$ signaling pathway plays an essential role in the embryonic development and homeostasis of diverse adult tissues, and its deregulation has been implicated in the tumorigenesis and metastasis of various malignancies including breast cancer. Aberrant activation of the Hh pathway includes the following mechanisms: (I) Hh ligand-independent mechanism - Loss of function mutations in the Hh receptor Patched 1 (PTCH1) or gain of function mutations in the Smoothened (SMO) lead to constitutive activation of this pathway; (II) Autocrine signalingHh ligand produced by tumor cells stimulates the $\mathrm{Hh}$ signaling in tumor cells; (III) Paracrine signaling - tumor cell produced-Hh ligand activates stromal and endothelial cells that produce growth factors in microenvironment for supporting tumor growth and survival; and (IV) Reverse paracrine signaling - Hh ligand produced by stromal cells support tumor growth and survival. Upon the pathway activation, the Gli transcription factors, effectors of the $\mathrm{Hh}$ signaling, activate or inhibit transcription by binding to their responsive genes and interacting with the transcriptional complex. The Gli transcription factor family includes Gli1, Gli2, and Gli3 (1). Gli1 is a transcriptional activator whose expression has been recognized as an activation state of the Hh signaling pathway, Gli2 is either an activator or repressor, and Gli3 is a strong repressor of transcriptional activities. To date, a ligand-dependent autocrine model of activating the Hh signaling has been described in breast cancer, and both an autocrine and paracrine mechanisms in colorectal cancer, pancreatic cancer and prostate cancer $(2,3)$. Notably, a ligand-independent mechanism (mutations in PTCH1 and SMO) of the signaling has been well demonstrated in basal cell carcinoma and medulloblastoma $(4,5)$.

This has rendered the Hh pathway an attractive therapeutic target in cancer in recent years. Drugs that specifically target hedgehog signaling are being actively developed and tested in clinical trials (6). Vismodegib (GDC0449), a small molecule inhibitor of SMO, has recently received expedited approval for use in the treatment of advanced basal cell carcinoma by the United States Food and Drug Administration (http://www.fda.gov/Drugs/ InformationOnDrugs/ApprovedDrugs/ucm289571.htm). Agents inhibiting the Hh pathway are also being tested in women with advanced breast cancer in early clinical trials.

In the current issue of Chinese Fournal of Cancer Research, $\mathrm{Li}$ and colleagues have demonstrated a potentially promising clinical correlation between the recurrence-free survival (RFS) and the nuclear expression of Gli1 in breast tumors in 284 patients with stage I, II and III breast cancer derived from a single institution by immunohistochemistry (7). This association reached statistical significance by univariate analysis. Expression of Gli1 is localized in the malignant nuclei and cytoplasm, and is more abundant in breast tumor cells than adjacent non-malignant breast tissues. The authors also presented an intriguing observation that Glilexpression in the cancer interstitial tissues of the relapsed group was much higher than the non-relapsed group. It is speculated that Gli expression in the cancer interstitial tissues (tumor microenvironment or tumor stroma) is a result of activation of the receptor PTCH of 
the Hh pathway in stromal cells initiated by the tumor cell derived Hh ligand. This finding may suggest that the paracrine Hh signaling mechanism plays a role in breast cancer as it does in colorectal, prostate and pancreatic cancers. This, however, needs examination in preclinical models and further translational research to evaluate the role of Gli1 in tumor progression.

In brief, this investigation has pointed out the potential importance of the Hh signaling pathway in breast cancer. If this pathway continues to be of interest and targeting the $\mathrm{Hh}$ signaling would be successful in cancers besides in basal cell carcinoma and medulloblastoma, robust standardization and validation of Gli1 immunohistochemistry procedure will be necessary. Preclinical evaluation of the Hh signaling pathway, particularly in animal models, could aid in further elucidating the activation mechanisms of the Hh signaling pathway in breast cancer. The correlation of Gli1 expression with clinical outcome in a larger breast cancer patient cohort will help in determining its independent prognostic value.

\section{Acknowledgements}

Disclosure: The authors declare no conflict of interest.

Cite this article as: Takebe N, Hunsberger S, Yang SX. Expression of Gli1 in the hedgehog signaling pathway and breast cancer recurrence. Chin J Cancer Res 2012;24(4):257258. DOI: $10.3978 /$ j.issn.1000-9604.2012.09.07

\section{References}

1. Hui CC, Angers S. Gli proteins in development and disease. Annu Rev Cell Dev Biol 2011;27:513-37.

2. Kubo M, Nakamura M, Tasaki A, et al. Hedgehog signaling pathway is a new therapeutic target for patients with breast cancer. Cancer Res 2004;64:6071-4.

3. Sahebjam S, Siu LL, Razak AA. The utility of hedgehog signaling pathway inhibition for cancer. Oncologist 2012;17:1090-9.

4. Von Hoff DD, LoRusso PM, Rudin CM, et al. Inhibition of the hedgehog pathway in advanced basal-cell carcinoma. N Engl J Med 2009;361:1164-72.

5. Rudin CM, Hann CL, Laterra J, et al. Treatment of medulloblastoma with hedgehog pathway inhibitor GDC0449. N Engl J Med 2009;361:1173-8.

6. Takebe N, Harris PJ, Warren RQ, et al. Targeting cancer stem cells by inhibiting Wnt, Notch, and Hedgehog pathways. Nat Rev Clin Oncol 2011;8:97-106.

7. Li YH, Gao HF, Wang Y, et al. Overexpression of Gli1 in cancer interstitial tissues predicts early relapse after radical operation of breast cancer. Chin J Cancer Res 2012;24:263-74. 